\title{
Telehealth Uptake into Prenatal Care and Provider Attitudes during the COVID-19 Pandemic in New York City: A Quantitative and Qualitative Analysis
}

\author{
Nigel Madden, MD ${ }^{1} \quad$ Ukachi N. Emeruwa, MD, MPH ${ }^{1} \quad$ Alexander M. Friedman, MD, MPH ${ }^{1}$ \\ Janice J. Aubey, MD, MPH ${ }^{1}$ Aleha Aziz, MD, MPH ${ }^{1}$ Caitlin D. Baptiste, MD ${ }^{1}$ Jaclyn M. Coletta, MD ${ }^{1}$ \\ Mary E. D’Alton, MD ${ }^{1}$ Karin M. Fuchs, MD, MHA ${ }^{1}$ Dena Goffman, MD ${ }^{1}$ \\ Cynthia Gyamfi-Bannerman, MD, MS ${ }^{1}$ Sneha Kondragunta, MD ${ }^{1} \quad$ Nicole Krenitsky, MD, MBA ${ }^{1}$ \\ Russell S. Miller, MD ${ }^{1}$ Chia-Ling Nhan-Chang, MD ${ }^{1}$ Ashanda M. Saint Jean, MD ${ }^{1}$ \\ Hemangi P. Shukla, DO, MS ${ }^{1}$ Lynn L. Simpson, MD ${ }^{1}$ Erica S. Spiegel, MS ${ }^{1}$ Hope S. Yates, JD, MPH ${ }^{1}$ \\ Noelia Zork, MD ${ }^{1}$ Samsiya Ona, MD ${ }^{1}$
}

\author{
1 Department of Obstetrics and Gynecology, NewYork Presbyterian/ \\ Columbia University Irving Medical Center, Columbia University, \\ New York, New York \\ Am J Perinatol 2020;37:1005-1014.
}

Address for correspondence Alexander Friedman, MD, MPH, Division of Maternal-Fetal Fetal Medicine, Department of Obstetrics and Gynecology, Columbia University College of Physicians and Surgeons, 622 West 168th Street, New York, NY 10032

(e-mail: amf2104@cumc.columbia.edu).

\section{Abstract}

Keywords

- telehealth

- COVID-19

- health services

- prenatal care
Objective This study aimed to (1) determine to what degree prenatal care was able to be transitioned to telehealth at prenatal practices associated with two affiliated hospitals in New York City during the novel coronavirus disease 2019 (COVID-19) pandemic and (2) describe providers' experience with this transition.

Study Design Trends in whether prenatal care visits were conducted in-person or via telehealth were analyzed by week for a 5-week period from March 9 to April 12 at Columbia University Irving Medical Center (CUIMC)-affiliated prenatal practices in New York City during the COVID-19 pandemic. Visits were analyzed for maternal-fetal medicine (MFM) and general obstetrical faculty practices, as well as a clinic system serving patients with public insurance. The proportion of visits that were telehealth was analyzed by visit type by week. A survey and semistructured interviews of providers were conducted evaluating resources and obstacles in the uptake of telehealth.

Results During the study period, there were 4,248 visits, of which approximately onethird were performed by telehealth $(n=1,352,31.8 \%$ ). By the fifth week, $56.1 \%$ of generalist visits, $61.5 \%$ of MFM visits, and $41.5 \%$ of clinic visits were performed via telehealth. A total of 36 providers completed the survey and 11 were interviewed. Accessing technology and performing visits, documentation, and follow-up using the telehealth electronic medical record were all viewed favorably by providers. In transitioning to telehealth, operational challenges were more significant for health clinics than for MFM and generalist faculty practices with patients receiving public insurance experiencing greater difficulties and barriers to care. Additional resources on the patient and operational level were required to optimize attendance at in-person and video visits for clinic patients. received

May 8, 2020

accepted after revision

May 9, 2020

published online

June 9, 2020
Copyright $\odot 2020$ by Thieme Medical Publishers, Inc., 333 Seventh Avenue, New York, NY 10001, USA. Tel: +1(212) 760-0888.
DOI https://doi.org/

10.1055/s-0040-1712939. ISSN $0735-1631$. 
Conclusion Telehealth was rapidly implemented in the setting of the COVID-19 pandemic and was viewed favorably by providers. Limited barriers to care were observed for practices serving patients with commercial insurance. However, to optimize access for patients with Medicaid, additional patient-level and operational supports were required.

\section{Key Points}

- Telehealth uptake differed based on insurance.

- Medicaid patients may require increased assistance for telehealth.

- Quick adoption of telehealth is feasible.

The World Health Organization (WHO) declared the novel coronavirus disease 2019 (COVID-19) a public health emergency of international concern on January 30, 2020 and by March 11, 2020, the WHO officially classified it as a pandemic. ${ }^{1,2}$ New York City quickly became the epicenter of the COVID-19 pandemic, prompting the New York State governor to declare a disaster emergency and sign an executive order limiting gatherings and closing businesses. ${ }^{3}$ For obstetric patients receiving prenatal care in the greater New York City area, attending in-person prenatal care visits, including travel to and from appointments and interaction with health care personnel and staff, represented an additional potential source of exposure.

Telehealth in the form of video visits represents one approach to mitigating risk of viral exposure for patients and providers, and has been proposed as a mean of ensuring continued care in the setting of disasters. ${ }^{4,5}$ For a variety of medical specialties, telehealth may represent a means of maintaining patient care access in the setting of COVID-19 while facilitating physical distancing and lightening the burden of clinical practice settings. ${ }^{5}$ Prior research on integration of telehealth into the health care system has been conducted in rural communities and has demonstrated both challenges and opportunities for improved care. ${ }^{6,7}$

Given that many other locales in the United States may be faced with similar challenges related to the COVID-19 pandemic and that COVID-19 may recur seasonally, or some other disaster may require similar changes to health care delivery, the purpose of this study was to review adoption of telehealth for obstetric patients in a tertiary referral hospital and clinic system in New York City.

\section{Materials and Methods}

Columbia University Irving Medical Center (CUIMC)-affiliated obstetric ambulatory prenatal care facilities located in midtown Manhattan, Washington Heights in Upper Manhattan, Rockland County, and Westchester transitioned to telehealth for prenatal care during the COVID-19 outbreak in the greater New York City metropolitan area. These facilities provide care for patients with both Medicaid and commercial insurance. Patients accessing prenatal care at these sites deliver primarily at NewYork Presbyterian Morgan Stanley Children's Hospital of New York, a tertiary referral center performing approximately 4,600 deliveries per year, and NewYork Presbyterian/The Allen Hospital, a community hospital performing approximately 2,300 deliveries per year. This study was approved by the Columbia University Irving Medical Center review board (AAAS9987).

Telehealth was incorporated into prenatal care at an accelerated rate beginning from March 16, 2020, shortly after schools, bars, and restaurants in New York City were ordered to close. Video visits were performed with Epic Haiku and Canto with patients accessing care via the Epic Connect and MyChart application on their phones or other portable devices (Epic, Verona, WI). Providers were trained in this software the week prior to adoption via a short video tutorial course ( $\sim 15$ minutes in duration) and user guide with additional support as needed. Patients were guided through installation of software by staff either by in-person visit, by e-mailed instructions, and/or remotely by telephone guidance.

To understand the degree to which telehealth was utilized in the setting of the COVID-19 pandemic, we examined the proportion of attended prenatal care visits that were able to be performed remotely over a 5-week period from March 9, 2020 to April 12, 2020, during which time telehealth was adopted across clinical sites. This study period included 1 week of preimplementation data. We evaluated prenatal visits for the following three types of practices providing prenatal care: (1) a generalist obstetrics and gynecology faculty practice providing care to patients with commercial insurance in Midtown Manhattan, Upper Manhattan, and Rockland County; (2) a maternal-fetal medicine (MFM) obstetrics and gynecology faculty practice providing care to patients with commercial insurance in Midtown Manhattan, Upper Manhattan, and Westchester; and (3) a clinic system in Upper Manhattan providing prenatal care to a population with Medicaid insurance primarily residing in Upper Manhattan and the Bronx which consolidated to a single site during the pandemic. The proportion of prenatal care visits performed by telehealth each week was determined. Additionally, whether visits were performed by telehealth was further analyzed based on whether visits were (1) a first prenatal care visit, (2) a return 
prenatal care visit, (3) a postpartum visit, (4) a consultation during pregnancy, or (5) a preconception consultation. In addition to the three practices, consultations for genetic counseling were also evaluated. Finally, the number of noshow visits was analyzed during this time period by practice, based on whether visits were scheduled to be held in-person or via telehealth. In rare cases where telehealth visits were conducted by telephone and not video because a technical failure occurred, these visits were categorized as telehealth visits. Categorical comparisons were performed with the Chisquare test or Fisher's exact test as appropriate with the Cochran-Armitage test for trend used to compare the proportion of telehealth visits by week. All statistical analyses were performed using SAS version 9.4 (SAS Institute, Cary, NC.).

In addition to the quantitative analysis of telehealth visits, a survey of providers routinely providing prenatal care was performed. Providers were also invited to perform a related semistructured interview. Investigators contacted potential respondents by e-mail to present the study and solicit their voluntary participation in the 3- to 5-minute survey and a related 10 - to 15 -minute interview. All providers who conducted telehealth visits during the implementation period were eligible to participate in both the survey and interviews. The survey consisted of a demographic and background questionnaire followed by a two-part set of questions designed to ascertain provider experience and satisfaction with the integration of telehealth. Part one of the survey comprised a series of statements to which each provider noted their agreement on a 5-point Likert's scale. Part two consisted of a short series of multiple-choice questions designed to better understand specifics surrounding the process of operationalization of telehealth. The survey was developed and housed electronically in the REDCap database. To further characterize the impact of telehealth uptake on our Medicaid population, we performed a sensitivity analysis looking at the survey results and perceptions among health clinic providers only.

For the interview portion, participants were informed about the purpose of the study and verbal consent was obtained to perform, record, and transcribe the interview prior to beginning. Each individual was asked a series of open-ended questions regarding their specific experiences with integration of telehealth into their practice. The recordings were transcribed, deidentified, and stored in the REDCap database. Providers who were operations leaders in both the MFM and generalist faculty practices and health clinics were additionally queried regarding telehealth transition and uptake. Given the COVID-19 pandemic, most interviews were conducted using Zoom meetings with the Voice Memos application when conducted in person. Each interview record was transcribed verbatim and reviewed by a group of three authors (S.O., U.E., and N.M.) for accuracy. The qualitative data were evaluated using a framework approach with thematic analysis. ${ }^{8}$ The transcribed interview responses were used to generate broad categories. Three investigators (S.O., U.E., and N.M.) assessed the responses for divergence and convergence, weighting each theme by assessing the frequency of it being mentioned.

\section{Results}

During the study period, there were 4,248 pregnancy-related ambulatory visits of which 950 (22.4\%) were to generalists, 980 (23.1\%) were to MFM providers, and 2,318 (54.6\%) were to clinics ( - Table 1). Approximately one-third of visits were performed by telehealth during the study period ( $n=1,352,31.8 \%)$. For genetic counseling appointments, two-thirds of visits took place via telehealth (96/143, $67.1 \%)$. Over the study period, the proportion of telehealth visits increased significantly for each practice $(p<0.01$ for all based on the Cochran-Armitage test for trend). By the fifth week, $56.1 \%$ of visits in the generalist faculty practice, $61.5 \%$ of visits in the MFM faculty practice, $41.5 \%$ of health clinic visits, and $100 \%$ of genetic counseling visits were performed via telehealth ( - Fig. 1); the increased use of telehealth in the generalist and MFM faculty practices compared with the clinics was statistically significant ( $p<0.01$ based on the Cochran-Armitage test for trend). Analyzing telehealth utilization by visit type, by the last week of the study period postpartum visits were most likely to be performed via telehealth (87.3\%), followed by return prenatal visits (47.8\%), and new prenatal visits (29.3\%; -Fig. 2). Analyzing visit types by practice in the last week of the study period, MFM was significantly more likely to perform new prenatal visits $(81.3 \%)$ by telehealth compared with generalists (35.3\%) and the health clinics (18.1\%; $p<0.01$; - Figs. $3 \mathrm{~A}-\mathrm{C}$ ). For return prenatal visits during the final week of the study, $53.6 \%$ were performed via telehealth for the MFM faculty practice compared with $58.0 \%$ for the generalist faculty practice, and $41.6 \%$ for clinics $(p<0.01)$. For postpartum visits at the final week of the study, $85.7 \%$ were performed via telehealth at health clinics, $96.7 \%$ at the MFM faculty practice, and $57.1 \%$ at the faculty generalist practice $(p<0.01)$. Evaluating appointment no-shows, at no point in the study period were no-show rates above $10 \%$ for the generalist or MFM faculty practices for either in-person or telehealth visits. In comparison, for the health clinics $24.1 \%$ of visits were no-shows in week 1 , rising to $47.4 \%$ in week 3 , and then decreasing to $25.8 \%$ in week 5 ( - Fig. 4 AC). The proportion of no-show appointments for telehealth versus inpatient visits was similar at health clinic appointments weeks 3 through 5 .

For the survey, 36 of 68 queried (53\%) providers caring for prenatal patients provided survey information. The majority of respondents were physicians $(n=30,83.3 \%)$, half of whom $(n=18)$ were attending physicians, and a quarter of whom were MFM fellows or attendings (- Supplementary Table S1, available in the online version). Overall, $97 \%$ of respondents believed telehealth increased access for patients and 92\% believed that telehealth provided adequate care when appropriately scheduled (-Fig. 5).The majority of providers believed telehealth was convenient both for their patients and for their practice. While only $45 \%$ of providers were motivated to use telehealth prior to the pandemic, $89 \%$ would continue using the technology in the future. The majority of providers (80\%) said that telehealth technology was easy to set up and 56\% felt support was adequate during transition 


\begin{tabular}{|c|c|c|c|c|c|c|c|c|c|c|c|c|c|c|c|}
\hline \multirow{2}{*}{$\begin{array}{l}\text { Practice } \\
\text { Study week }\end{array}$} & \multicolumn{5}{|c|}{ Generalist obstetricians } & \multicolumn{5}{|c|}{ Maternal-fetal medicine } & \multicolumn{5}{|c|}{ Health clinics } \\
\hline & 1 & 2 & 3 & 4 & 5 & 1 & 2 & 3 & 4 & 5 & 1 & 2 & 3 & 4 & 5 \\
\hline \multicolumn{16}{|l|}{ Attended visits } \\
\hline All attended scheduled visits & 227 & 168 & 180 & 177 & 198 & 190 & 156 & 163 & 237 & 234 & 520 & 442 & 304 & 473 & 579 \\
\hline Telehealth visits & 0 & 23 & 82 & 97 & 111 & 4 & 43 & 96 & 152 & 144 & 0 & 62 & 101 & 197 & 240 \\
\hline $\begin{array}{l}\text { All preconception } \\
\text { consultations }\end{array}$ & 1 & 1 & 4 & 2 & 0 & 6 & 2 & 1 & 1 & 1 & 1 & 0 & 0 & 0 & 0 \\
\hline $\begin{array}{l}\text { Telehealth preconception } \\
\text { consultations }\end{array}$ & 0 & 1 & 4 & 2 & 0 & 0 & 1 & 1 & 1 & 1 & 0 & 0 & 0 & 0 & 0 \\
\hline All return OB prenatal visits & 181 & 136 & 154 & 151 & 174 & 148 & 133 & 118 & 180 & 179 & 390 & 350 & 249 & 395 & 454 \\
\hline $\begin{array}{l}\text { Telehealth return } \mathrm{OB} \\
\text { prenatal visits }\end{array}$ & 0 & 17 & 67 & 81 & 101 & 1 & 35 & 65 & 107 & 96 & 0 & 48 & 85 & 162 & 189 \\
\hline All new OB prenatal visits & 24 & 19 & 9 & 12 & 17 & 14 & 13 & 28 & 33 & 16 & 92 & 65 & 37 & 46 & 83 \\
\hline $\begin{array}{l}\text { Telehealth new OB } \\
\text { prenatal visits }\end{array}$ & 0 & 0 & 2 & 3 & 6 & 0 & 6 & 19 & 25 & 13 & 0 & 2 & 4 & 8 & 15 \\
\hline $\begin{array}{l}\text { All consultations } \\
\text { during pregnancy }\end{array}$ & 0 & 0 & 0 & 0 & 0 & 5 & 3 & 6 & 3 & 8 & 0 & 0 & 0 & 0 & 0 \\
\hline $\begin{array}{l}\text { Telehealth consultations } \\
\text { during pregnancy }\end{array}$ & 0 & 0 & 0 & 0 & 0 & 0 & 0 & 4 & 2 & 5 & 0 & 0 & 0 & 0 & 0 \\
\hline All postpartum visits & 21 & 12 & 13 & 12 & 7 & 17 & 5 & 10 & 20 & 30 & 37 & 27 & 18 & 32 & 42 \\
\hline Telehealth postpartum visits & 0 & 5 & 9 & 11 & 4 & 3 & 1 & 7 & 17 & 29 & 0 & 12 & 12 & 27 & 36 \\
\hline \multicolumn{16}{|l|}{ No-show visits } \\
\hline All no-shows & 5 & 10 & 9 & 7 & 6 & 2 & 3 & 6 & 9 & 17 & 165 & 186 & 274 & 227 & 201 \\
\hline Telehealth no-shows & 0 & 2 & 6 & 4 & 2 & 0 & 0 & 6 & 9 & 14 & 0 & 10 & 114 & 109 & 73 \\
\hline
\end{tabular}

Abbreviations: COVID-19. Novel coronavirus disease 2019; OB, obstetric.

Note: Number of visits by week for each of three practices is demonstrated: (1) generalist obstetrician gynecologists, (2) maternal-fetal medicine specialists, and (3) health clinics providing care to a primarily Medicaid population staffed by generalists, maternal-fetal medicine specialists, nurse practitioners, and nurse midwives.

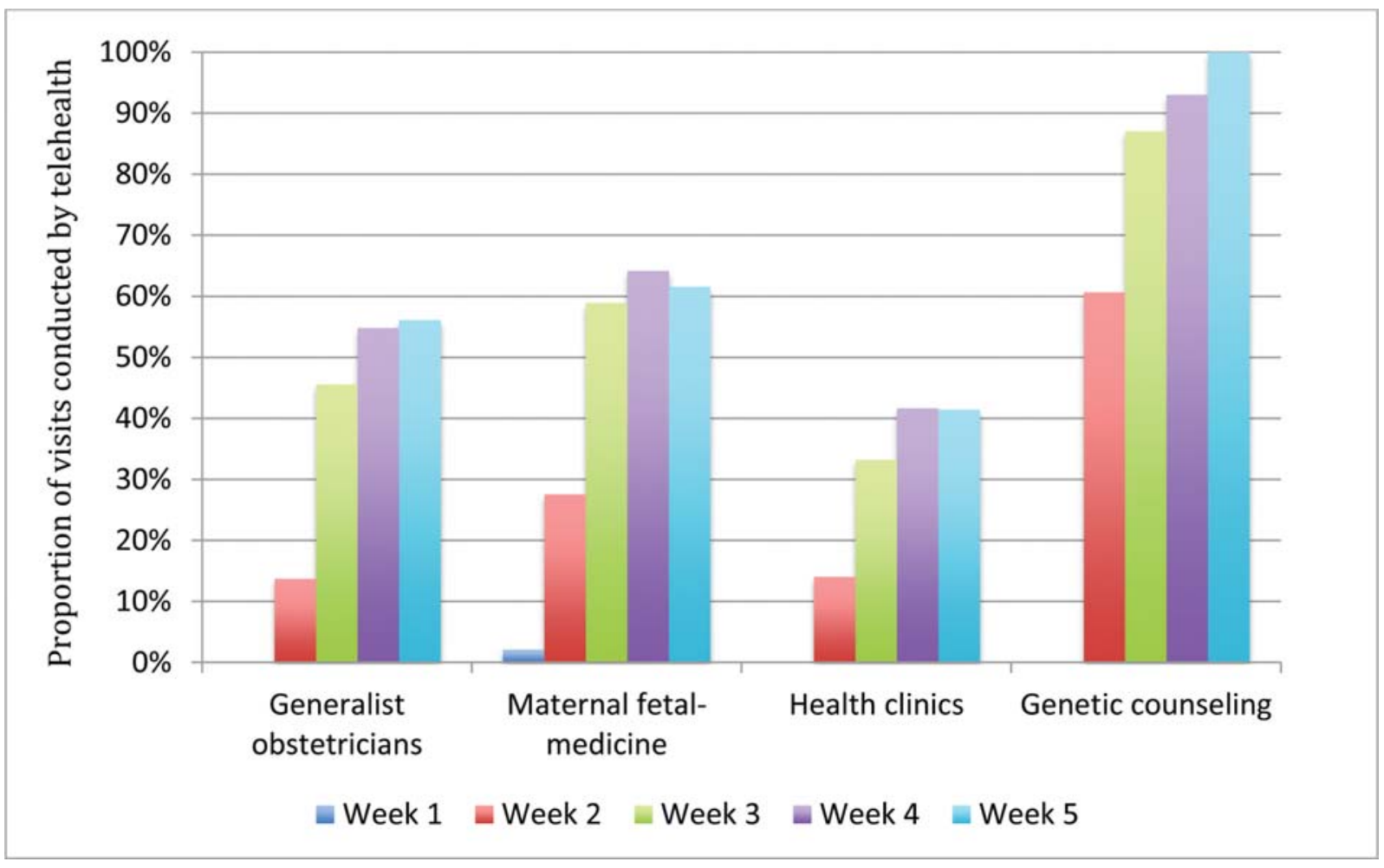

Fig. 1 Proportion of scheduled visits conducted by telehealth by practice group by week during the COVID-19 pandemic. The figure demonstrates the proportion of outpatient obstetrical visits conducted by telehealth for generalist obstetrician gynecologists, maternal-fetal medicine specialists, health clinics, and genetic counselors by week over a 5-week period from March 9, 2020 through April 12, 2020. 


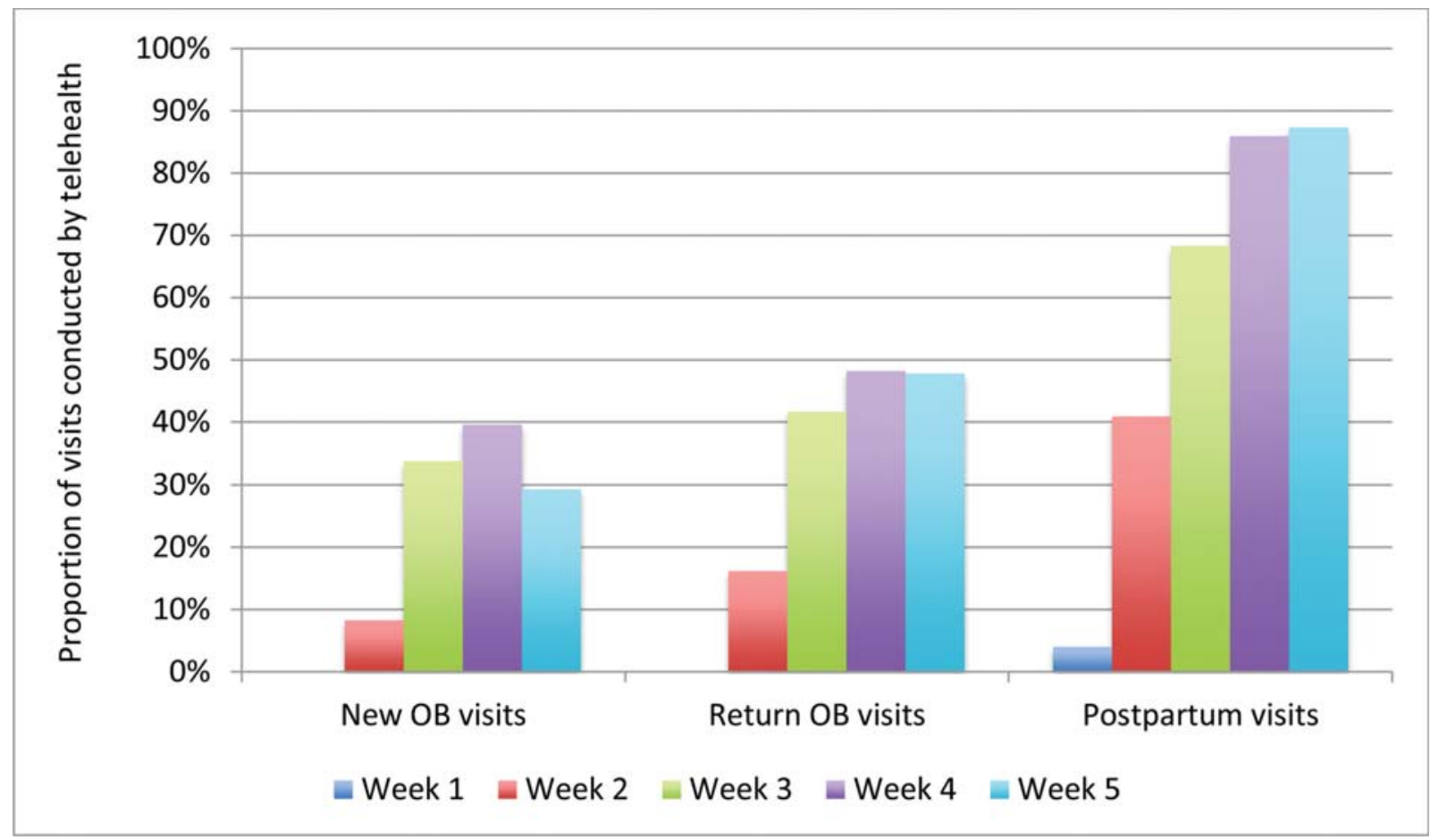

Fig. 2 Proportion of scheduled visits conducted by telehealth by week by type of visit during the COVID-19 pandemic. The figure demonstrates the proportion of outpatient obstetrical visits conducted by telehealth for new prenatal care visits, return prenatal care visits, and postpartum visits by week over a 5-week period from March 9, 2020 through April 12, 2020. COVID-19, novel coronavirus 2019; OB, obstetrician.
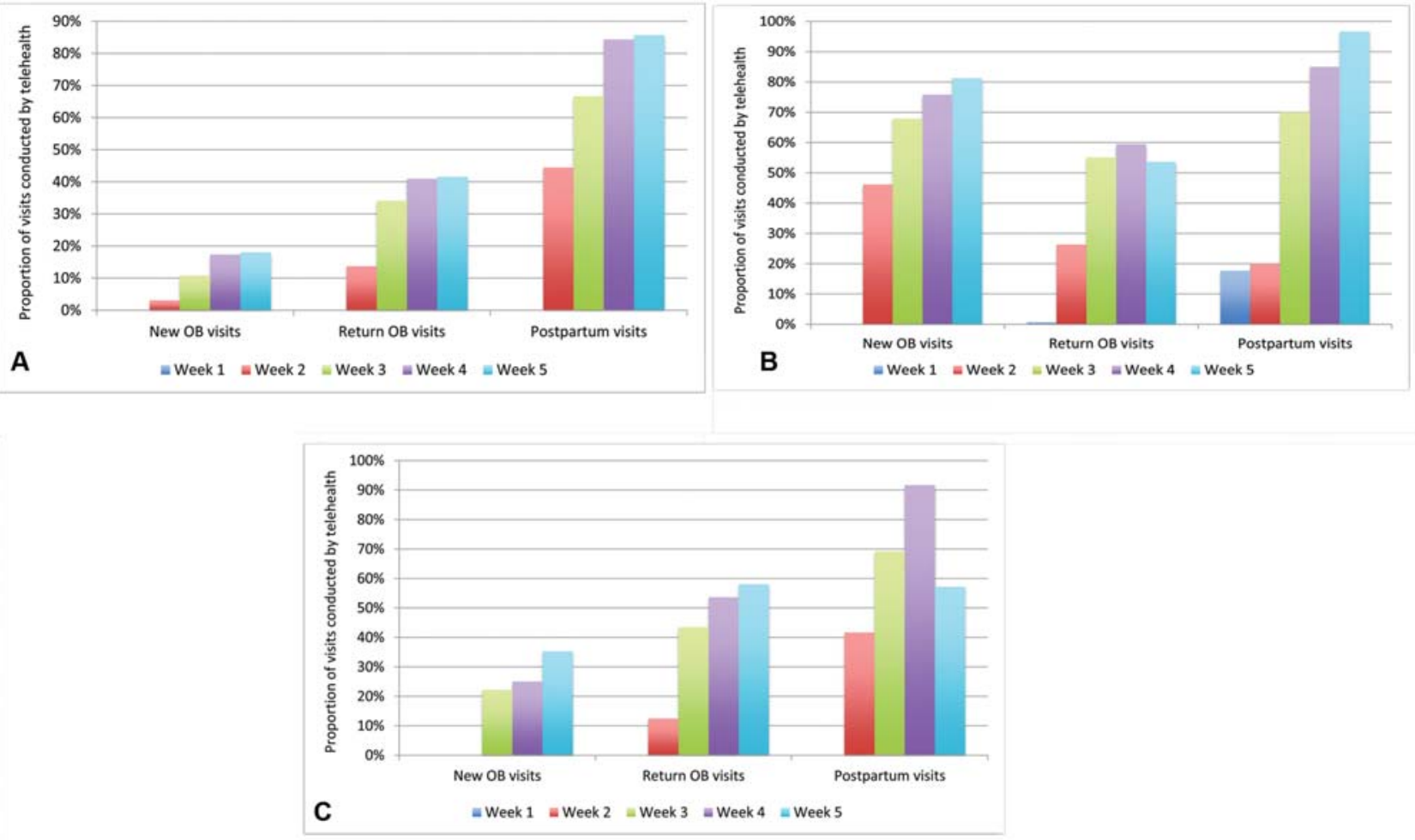

Fig. 3 (A) Proportion of scheduled visits conducted by telehealth at health clinics by week by type of visit. (B) Proportion of scheduled visits conducted by telehealth by MFM providers by week by type of visit. (C) Proportion of scheduled visits conducted by telehealth by generalist obstetrician-gynecologists by week by type of visit. The figure demonstrates the proportion of outpatient obstetrical visits conducted by telehealth for each type of obstetrical visit by week over a five week period from March 9, 2020 through April 12, 2020 individually for health 

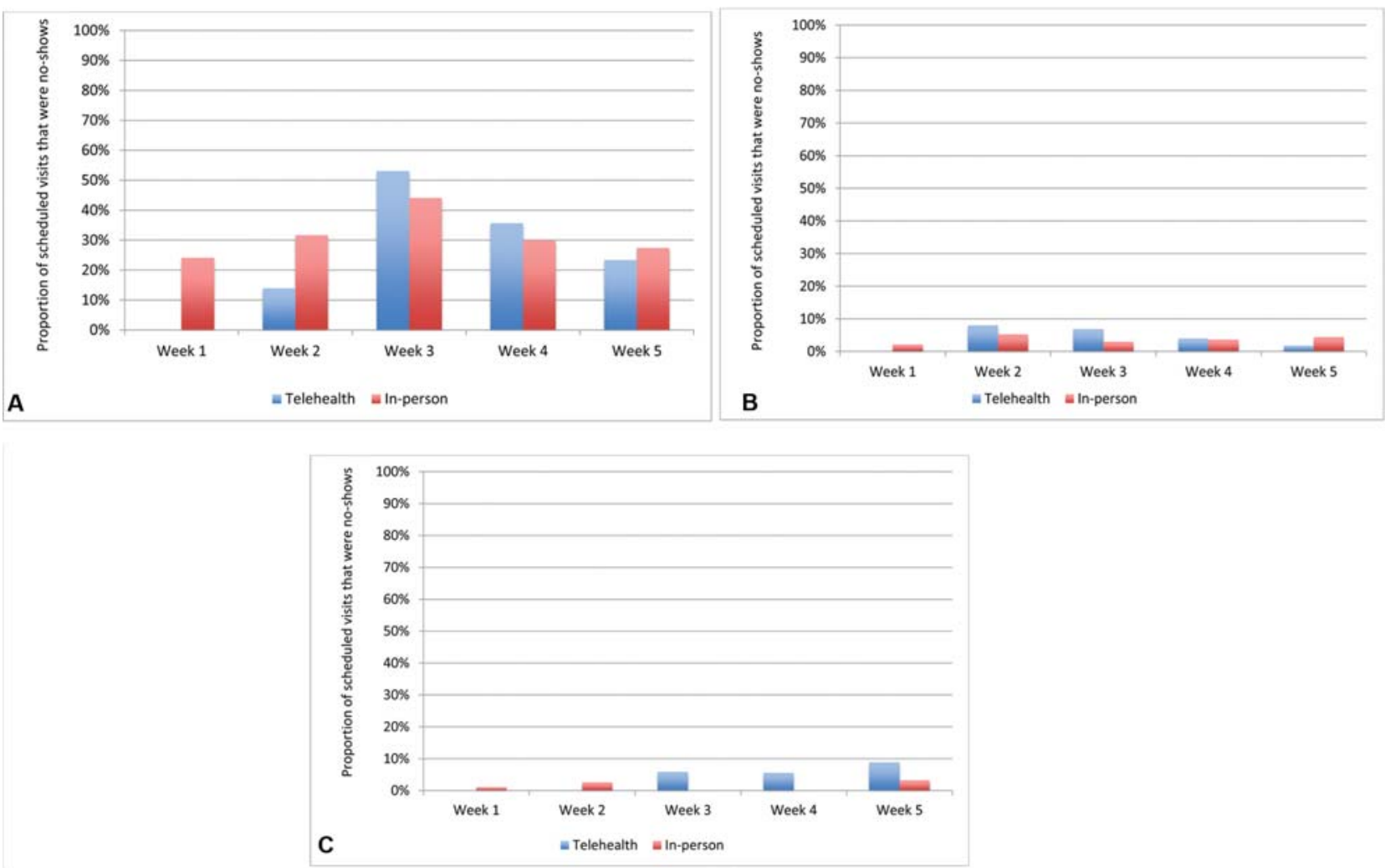

Fig. 4 (A) Proportion of visits that were no-shows for telehealth versus in-person visits at health centers by week. (B) Proportion of visits that were no-shows for telehealth versus in-person visits at generalist practices by week. (C) Proportion of visits that were no-shows for telehealth versus in-person visits at MFM practices be week. The figure demonstrates the proportion of outpatient obstetrical visits scheduled for telehealth visits and in-person visits that were no-shows by week from March 9, 2020 through April 12, 2020 for health center visits (A), generalist practices (B), and MFM practices (C). MFM, maternal-fetal medicine.

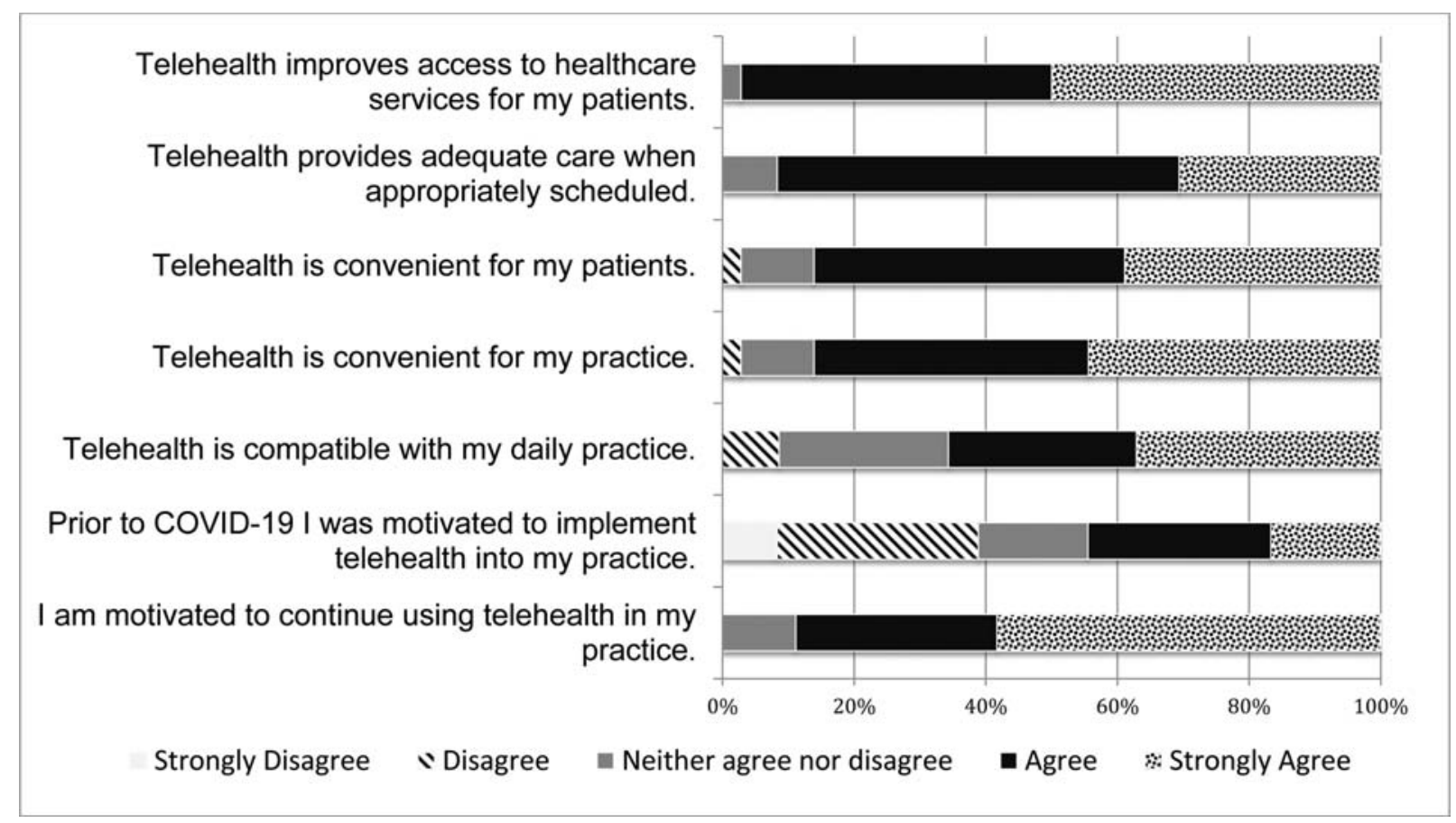

Fig. 5 Prenatal provider attitudes toward telehealth. This figure demonstrates provider degree of agreement with seven survey statements designed to evaluate attitudes and beliefs to toward the use of telehealth in clinical practice on a 5-point Likert's scale. COVID-19. Novel coronavirus disease 2019 . 


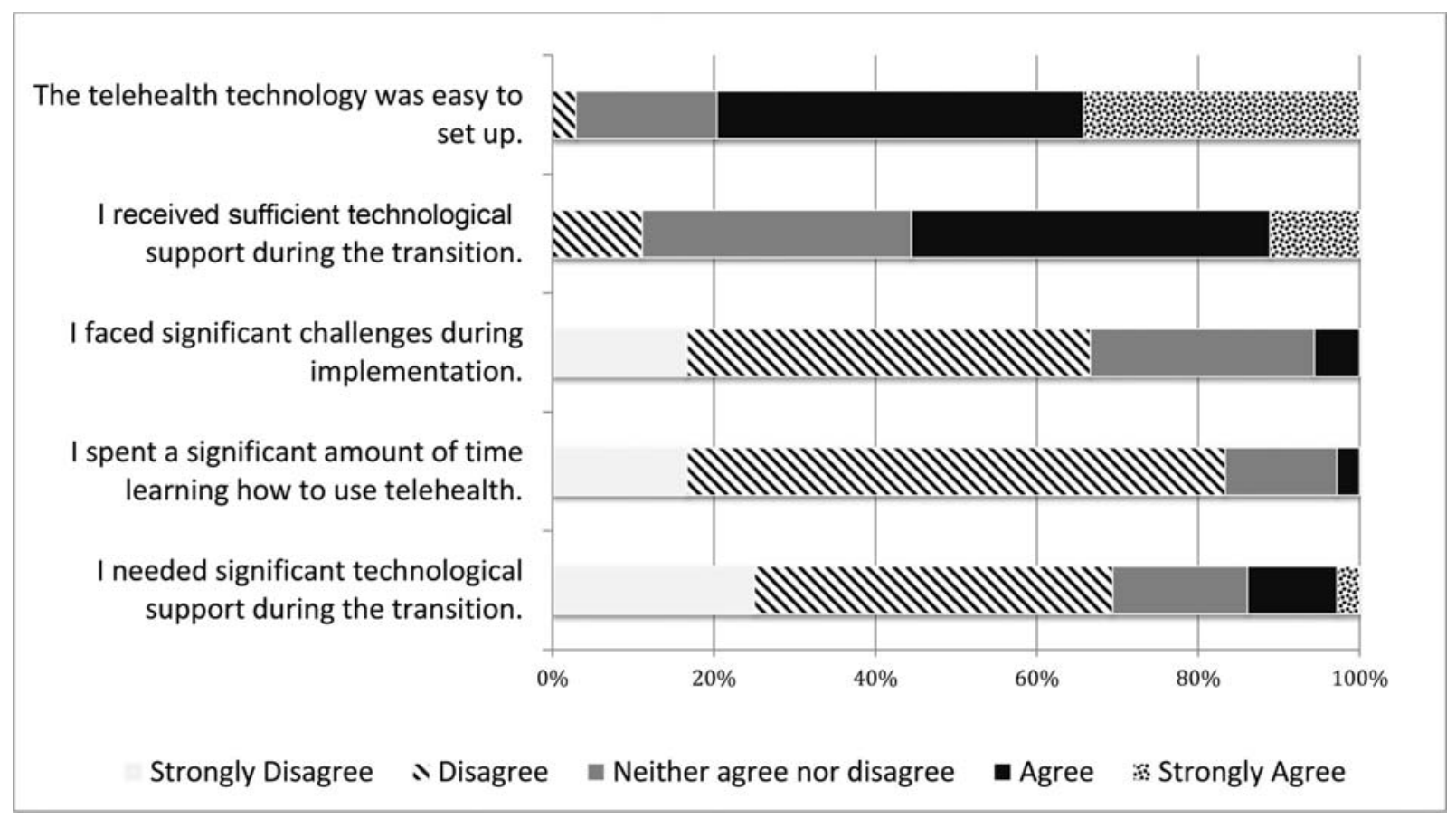

Fig. 6 Prenatal provider experience with telehealth implementation. This figure demonstrates provider degree of agreement with five survey statements designed to evaluate practitioner experience with the telehealth implementation process on a 5-point Likert's scale.

(-Fig. 6). Minorities of providers felt they experienced significant challenges during implementation (6\%) that they spent significant time on implementation (3\%), or that they needed significant technological support (14\%). In comparing video (and rare telephone) visits to in-person visits, pluralities or majorities of providers stated that video visits did not change preparation time (50\%), documentation time (56\%), ease of results of follow-up (69\%), patient rapport (53\%), billing difficulties (39\%), or patient safety (47\%; - Fig. 7). Providers were more likely to think that efficiency increased (42\%) than decreased (31\%) and that time of visit decreased (50\%) compared with increased (19.4\%) with telehealth. In evaluating barriers to care, the most common obstacle cited by providers (78\% of respondents) was patient difficulty in accessing and using technology. Resources cited by providers as helpful in telehealth

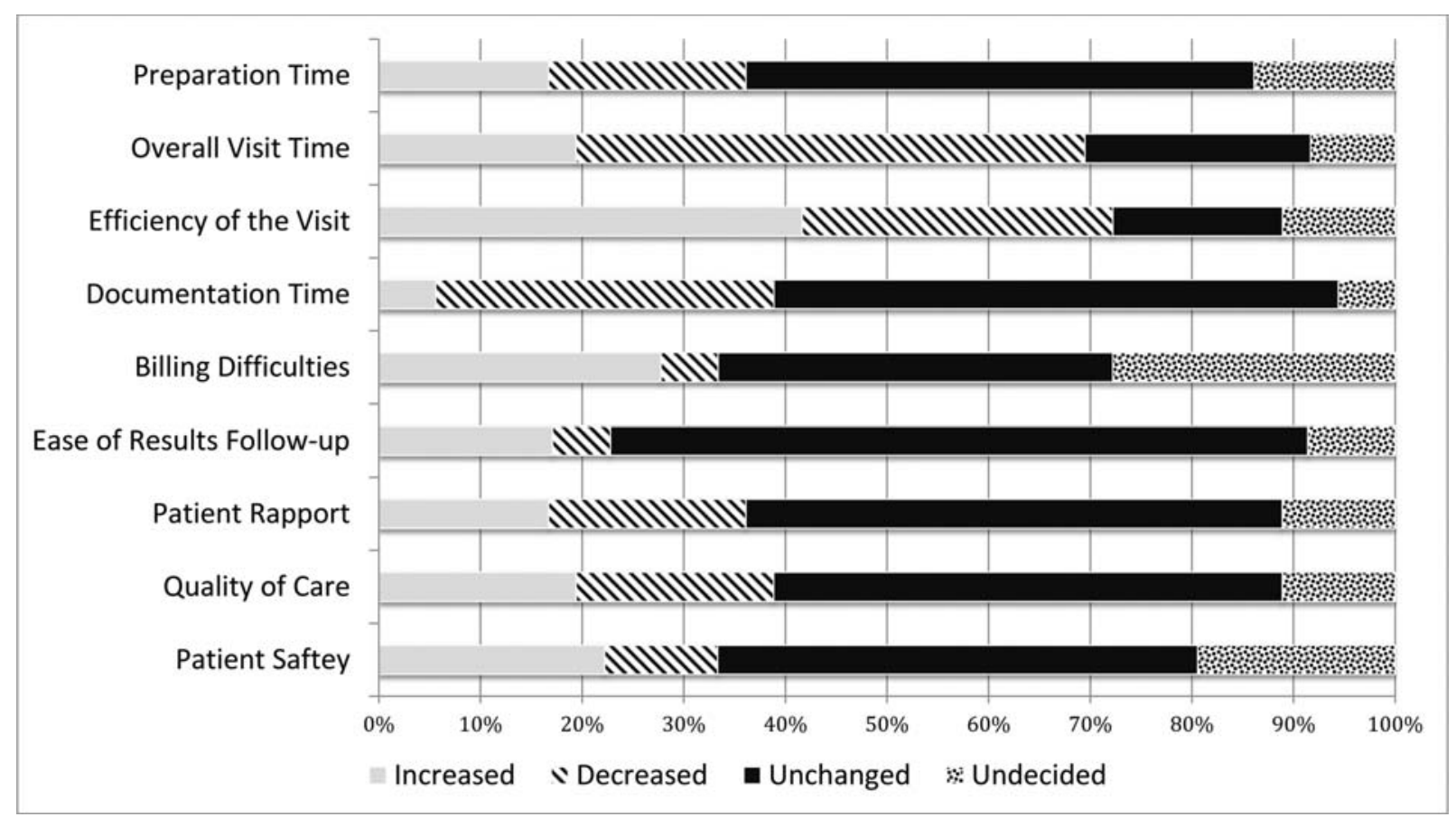

Fig. 7 Prenatal provider perceptions of characteristics of telehealth compared with in-person visits. This figure demonstrates provider perceptions about how telehealth has changed various visit metrics as compared with in-person visits. 
integration included administrative support staff (including members of the hospital information technology (IT) help center, Epic electronic medical record (EMR) help personnel, and volunteer staff; 67\%), departmental support (including modules and training videos provided by the department, provision of office space specifically suitable for telehealth, provision of mobile devices to staff whose devices were not compatible; 67\%), and support from IT (47\%). When the data were restricted to health clinic providers $(n=23)$, results were similar to the primary analysis (-Supplementary Figs. 1-3, available in the online version).

In performing interviews with 11 of 36 survey respondents (of whom 12 were invited for interviews), several key themes arose (-Table 2). Providers noted that patient benefits of telehealth included decreased COVID-19 exposure, that patients could continue to access care in the setting of stay-at-home instructions, and there were telehealth benefits for patients with increased childcare responsibilities. Telehealth was facilitated by patients having easy access to required technology and devices. Perceived barriers for patients attending health clinics included the following: (1) hesitation or anxiety in using telehealth, (2) difficulty in setting up software, and (3) accessing continuous Wi-Fi or data for the visit. In interviews, providers noted that integrating telehealth was facilitated by access to colleagues with telehealth experience, the ease of use of the technology, online modules and work flow documents for using the telehealth software and interface, and easy access to EMR data to plan visits in advance. A barrier to use noted by providers was that interpreter services were more cumbersome during telehealth visits. Departmental factors noted to facilitate care included guideline templates for prenatal care appointments and fetal testing and ultrasound indications.

In discussing telehealth transition in the clinics, provider leadership noted the following:

1. Additional office staff were required to rapidly enroll patients in Epic, so that telehealth visits could be performed.

2. Additional training for office staff was required specifically to schedule and manage telehealth appointments.

3. Determination of which visits could be performed via telehealth versus which mandated in-person visits was initially a time-intensive process.

4. Additional staff supervisors were required to manage these processes.

5. Provider workflow and scheduling had to be reorganized to facilitate telehealth visits.

6. Phone calls and text reminders within 48 hours of appointment time improved patient attendance for telehealth and in-person visits.

Table 2 Qualitative survey findings on the use of telehealth for prenatal care based on provider interviews

\begin{tabular}{|c|c|c|c|}
\hline & Benefits of telehealth & Facilitators of telehealth & Barriers to telehealth \\
\hline Patient & $\begin{array}{l}\text { - Limits COVID-19 exposure } \\
\text { - Ensures continued access to care } \\
\text { - Convenience for patients with in- } \\
\text { creased childcare responsibilities }\end{array}$ & $\begin{array}{l}\text { - Concerns of COVID-19 exposure } \\
\text { - Appreciation of continued care } \\
\text { - Access to required technology and } \\
\text { devices (i.e., smart phones, tablet, } \\
\text { e-mail) }\end{array}$ & $\begin{array}{l}\text { - Discomfort/hesitation/anxiety with } \\
\text { telehealth visits and technology } \\
\text { - Initial set-up technically difficult } \\
\text { - Technical difficulties with logging on } \\
\text { and maintaining continuous Wi-Fi or } \\
\text { data connection through visit } \\
\text { - Need for home monitoring devices } \\
\text { (i.e., fetal heart tone Doppler's, } \\
\text { blood pressure cuffs) }\end{array}$ \\
\hline Provider & $\begin{array}{l}\text { - Limits COVID-19 exposure } \\
\text { - Ensures continued access to care }\end{array}$ & $\begin{array}{l}\text { - Access to colleagues with prior tele- } \\
\text { health experience } \\
\text { - Ease of use of telehealth technology } \\
\text { - Online modules/work-flow documents } \\
\text { on how to use telehealth software and } \\
\text { interface } \\
\text { - Accessible EMR data to plan telehealth } \\
\text { care encounters in advance of visit }\end{array}$ & 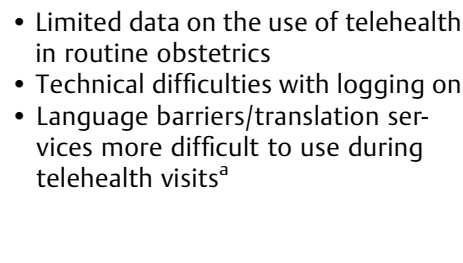 \\
\hline Clinic/Office & - Limits COVID-19 exposure & $\begin{array}{l}\text { - Protection of patients and staff } \\
\text { - Online modules for support staff } \\
\text { - Assistance for office staff in telehealth } \\
\text { scheduling and administration } \\
\text { - Centralized patient call center to facili- } \\
\text { tate patient technological trouble- } \\
\text { shooting and scheduling }\end{array}$ & $\begin{array}{l}\text { - Rapidity of integration } \\
\text { - Recent transition to EMR/unfamiliar- } \\
\text { ity with telehealth administration and } \\
\text { scheduling } \\
\text { - Lack of up-to-date patient contact } \\
\text { information a } \\
\text { - Additional support staff required } \\
\text { numbers } \\
\text { - } \text { Challenges with patient scheduling }^{\text {a }}\end{array}$ \\
\hline Departmental & & $\begin{array}{l}\text { - Development of guidelines regarding } \\
\text { which antenatal visits are appropriate } \\
\text { for telehealth } \\
\text { - Development of guidelines regarding } \\
\text { frequency and interval of ultrasound } \\
\text { monitoring }\end{array}$ & $\begin{array}{l}\text { - Rapid implementation precluded } \\
\text { small scale testing } \\
\text { - Compliance/billing issues }\end{array}$ \\
\hline
\end{tabular}

Abbreviations: COVID-19. Novel coronavirus disease 2019; OB, obstetric; EMR, electronic medical record.

${ }^{a}$ Noted by providers to be more common in the care of health clinic patients. 
7. Patient outreach and phone calls to perform troubleshooting enhanced telehealth use.

Significant barriers to telehealth and in-person visits included the following:

1. Many patients were fearful of COVID-19 infection and delayed or abstained from seeking medical attention.

2. The Epic MyChart interface did not include an option with Spanish instructions making enrollment more challenging for many patients.

Key features that facilitated successful use and transition to telehealth included the following:

1. A telehealth-enabled EMR that allowed simultaneous review of records and documentation during the visit.

2. Continuous IT assistance for providers and staff.

3. Holding regular meetings with a dedicated telehealth leadership team to review progress, troubleshoot problem areas, and review patient enrollment and scheduling.

\section{Discussion}

In this study of transition to prenatal telehealth during the COVID-19 pandemic in New York City, we were able to perform a large proportion of visits virtually. Uptake was rapid with approximately half of visits across sites conducted virtually by the week of March 30 to April 3, 2020. While providers generally had positive attitudes toward telehealth visits, a key finding from this analysis is that the transition to virtual prenatal care was more challenging for patients with Medicaid insurance receiving care at health clinics than for women with commercial insurance in generalist and maternal fetal medicine faculty practices. Factors related to differential care attendance included (1) operational considerations such as requiring increased staffing in clinics and (2) patient factors related to technological proficiency, language barriers, Wi-Fi and data access, child care, and fear of infection.

These findings have several implications for clinical management. First, because patients with Medicaid insurance may face additional barriers to implement telehealth, it may be reasonable to create in advance a framework for patient enrollment and operational changes prior to widespread telehealth adoption (-Table 3 ). While other clinical operations may be curtailed on a hospital or system level in the setting of the COVID-19 pandemic, obstetrical services including prenatal care must be continued to be offered at full capacity. Therefore, additional IT and administrative resources are likely required to optimize telehealth transition. This study found that many operational issues were able to be addressed in a relatively short time period in the setting of a major public health emergency; with more lead time and advanced planning, telehealth transition may be even less disruptive for patients with public insurance. Second, that a large proportion of patients missed in-person visits during the third week of the analysis (likely due to factors such as child care responsibilities and fear of infection) supports telehealth as a critical resource in the setting of the pandemic. While comparative effectiveness research on telehealth is limited outside the pandemic, ${ }^{9,10}$ current conditions support that there is a substantial benefit to telehealth use during the pandemic and beyond. Third, there was limited evidence of barriers to offering care from the standpoint of provider workflow. Accessing technology, performing visits, documentation, and follow-up using the telehealth EMR were all viewed favorably by providers. These findings support that appropriate telehealth can be operationalized for providers quickly. Finally, the low rate of missed appointments for private insurance patients supports that this population faces fewer challenges with accessing telehealth and may require less support.

Table 3 Recommendations regarding resources and management for telehealth implementation in the setting of the COVID-19 pandemic

1. Provider electronic medical record telehealth training resources

- Online tutorials

- Printed work-flow guidelines

- In-person electronic medical record support staff

2. Patient educational and administrative support

- Ensure up-to-date contact information for all patients

- Develop educational materials and instructions for patients in multiple languages

- Schedule patients in-person visit or remotely to set up telehealth technology prior to their first visit

- Facilitate access to required home monitoring devices including a blood pressure cuffs, pulse oximetry, and scales

3. Clinical leadership team

- Review scheduled visits to decide whether telehealth may be appropriate

- Develop a visit-type coding system to communicate with administrative information technology staff the needs of each patient for appropriate visit booking

4. Administrative leadership team

- Ensure all patients are scheduled appropriately based on needs and visit type (new prenatal visit, follow-up prenatal visit, postpartum, preconception counseling, prenatal consultation)

- Ensure patients have set up software prior to telehealth visits

- Instruct patients to call if they have COVID-19 symptoms prior to in-person visits

5. Departmental administrative resources

- Standardized billing approaches for telehealth visits

- Formalized guidelines for telehealth versus in-person prenatal visits

- Formalized guidelines for prenatal ultrasound and fetal testing

Abbreviation: COVID-19. Novel coronavirus disease 2019. 


\section{Limitations and Strengths}

There are some limitations in interpreting the findings of this study. First, we did not directly survey or interview patients, and inferences related to barriers to care are made indirectly based on provider responses and analyzing appointment data. We are not able to empirically determine to what degree appointments were not attended because of child care responsibilities, limited access to devices and connectivity, challenges with using telehealth software leading to conversion to voice only visits, administrative and scheduling issues, financial repercussions of the pandemic, or general fear and anxiety of attending appointments. Financial repercussions of the COVID19 pandemic may have also been a factor in both attending inperson and virtual visits by decreasing access to Wi-Fi and data plans for mobile devices. Second, we do not have information regarding how patients perceived telehealth prenatal care, and it is possible that they could view it differently than providers. Third, all of the findings related to this analysis are dependent on use of one EMR platform. It is possible that provider satisfaction and operational issues could be better or worse with different EMR products. Strengths of this study include that the analysis was based on real-time implementation of widespread telehealth in the setting of a major public health crisis, that we demonstrated usability and satisfaction among providers, and that major operational challenges and barriers for health clinic patients were able to be significantly addressed in real-time to improve patient access.

\section{Conclusion}

In conclusion, rapid transition to telehealth for prenatal care was feasible and associated with provider satisfaction in this study. Significant barriers to telehealth may be present for patients with Medicaid insurance that may require additional support to resolve.

\section{Funding}

None.

\section{Conflict of Interest}

M.E.D. has had a leadership role in ACOG II's Safe Motherhood Initiative which has received unrestricted funding from Merck for Mothers. C.G.B. disclosed receiving money paid to her institution from SMFM/AMAG. She also received funding from Sera Prognostics and various funds for medicolegal work. She also disclosed receiving $\mathrm{NIH}$ (National Institute of Health) grants. Russell Miller disclosed receiving honorarium for writing a chapter on TRAP sequence for UpToDate. He received funds for medicolegal consulting (cases entirely unrelated to the topic of this study). The other authors did not report any potential conflicts of interest.

\section{References}

1 WHO Director General's opening remarks at the media briefing on COVID-19. March 11, 2020. Available at: https://www.who.int/dg/ speeches/detail/who-director-general-s-opening-remarks-at-themedia-briefing-on-covid-19-11-march-2020. Accessed May 13, 2020

2 Director-General WHO. Statement on the second meeting of the International Health Regulations (2005) Emergency Committee regarding the outbreak of novel coronavirus (2019-nCoV). January 30, 2020. Available at: www.who.int/news-room/detail/30-012020-statement-on-the-second-meeting-of-the-internationalhealth-regulations-(2005)-emergency-committee-regarding-theoutbreak-of-novel-coronavirus-(2019-ncov). Accessed May 13, 2020

3 Office NYSGs. Governor Cuomo Signs the 'New York State on PAUSE' Executive Order. Available at: /www.governor.ny.gov/news/governor-cuomo-signs-new-york-state-pause-executive-order. Accessed May 13, 2020

4 Lurie N, Carr BG. The role of telehealth in the medical response to disasters. JAMA Intern Med 2018;178(06):745-746

5 Hollander JE, Carr BG. Virtually perfect? Telemedicine for Covid19. N Engl J Med 2020;382(18):1679-1681

6 Gagnon MP, Duplantie J, Fortin JP, Landry R. Implementing telehealth to support medical practice in rural/remote regions: what are the conditions for success? Implement Sci 2006;1:18

7 Seto E, Smith D, Jacques M, Morita PP. Opportunities and challenges of telehealth in remote communities: case study of the Yukon telehealth system. JMIR Med Inform 2019;7(04): e11353

8 Castleberry A, Nolen A. Thematic analysis of qualitative research data: Is it as easy as it sounds? Curr Pharm Teach Learn 2018;10 (06):807-815

9 Implementing Telehealth in Practice. Implementing telehealth in practice: ACOG committee opinion summary, number 798 . Obstet Gynecol 2020;135(02):493-494

10 DeNicola N, Grossman D, Marko K, et al. Telehealth interventions to improve obstetric and gynecologic health outcomes: a systematic review. Obstet Gynecol 2020;135(02):371-382 